\title{
Publisher's Note: Strain Engineering of the Band Gap of HgTe Quantum Wells Using Superlattice Virtual Substrates [Phys. Rev. Lett. 117, 086403 (2016)]
}

Philipp Leubner, Lukas Lunczer, Christoph Brüne, Hartmut Buhmann, and Laurens W. Molenkamp (Received 7 August 2017; published 18 August 2017)

DOI: 10.1103/PhysRevLett.119.079901

This Letter was published online on 19 August 2016 with a typographical error on page 4. On page 4, left-hand column, the thirteenth line should read as "at higher fields. The $n$-type carrier density at the onset of two carrier conductance (black arrow) is $n_{e}^{*}=5.5 \times 10^{10} \mathrm{~cm}^{-2}$ ". The Letter has been corrected as of 11 August 2017. The text is incorrect in the printed version of the journal. 